\title{
Heat Transfer Analysis of PCM Slurry Flow between Parallel Plates
}

\author{
Hamidreza Shabgard, Han Hu, Philipp A. Boettcher, Matthew McCarthy, and Ying Sun* \\ Department of Mechanical Engineering and Mechanics, Drexel University, \\ Philadelphia, PA 19104, USA
}

\begin{abstract}
A CFD analysis of melting of solid particles during sedimentation in their own melt is presented. The motion of the solid particles is determined using a Lagrangian approach, while hydrodynamics and heat transfer throughout the fluid are determined using a finite volume scheme. Particle and fluid motions are two-way coupled through moving solid-liquid interfaces, whose morphologies are determined from the local interfacial heat fluxes and tracked using a deforming grid. The accuracy of the model is verified using benchmark solutions of a single particle undergoing simultaneous melting and settling. The results show that the presence of solid-phase particles within the liquid enhances the heat transfer between the bulk fluid and the heating surfaces due to improved mixing, as well as the latent heat associated with phase change. Particle loadings corresponding to solid volume fractions of $3 \%-18 \%$ have been considered here, and it is found that the average wall Nusselt number increases linearly with volume fraction. An enhancement in the average wall Nusselt number of $100 \%$ as compared to a singlephase flow is achieved using a slurry with $18 \%$ solid particles by volume. Initial particle arrangement is found to have a minimal effect on the overall heat transfer. Additionally, for a fixed solid volume fraction, it is found that particle diameter does not strongly influence heat transfer enhancement.
\end{abstract}

*Corresponding Author. Tel.:+1-215-895-1373; fax: +1-215-895-1478; Email: YSun@ coe.drexel.edu

(C) 2016. This manuscript version is made available under the Elsevier user license http://www.elsevier.com/open-access/userlicense/1.0/ 
KEYWORDS: Slurry flow, Phase change materials, Arbitrary-Lagrangian-Eulerian method, Melting, Heat transfer 


\section{Introduction}

Solid-liquid phase change materials (PCMs) are characterized by large energy densities that enable them to absorb and release large amounts of thermal energy within a small temperature range. For decades, these features have been of interest in short- and long-term thermal energy storage systems. More recently, a new PCM-based technology has been emerged to improve the thermal performance of heat exchangers by developing two-phase heat transfer fluids comprising PCM particles mixed with a base fluid [1,2]. These two-phase slurries benefit from larger apparent specific heats compared to single-phase fluids and are suitable for transferring large amounts of heat without a dramatic temperature rise [3]. In addition to efficient heat transfer, PCM slurry fluids can also serve as thermal storage medium, thereby eliminating the need for separate heat transfer and storage media in thermal energy storage systems [4].

Optimal design of heat exchangers/thermal storage systems utilizing the PCM slurries requires a thorough understanding of interfacial transport phenomena occurring between the solid and liquid phases of the slurry. While experimental measurements of interphase interactions in particulate flows are challenging, numerical models with different levels of complexity have been developed for the analysis of such systems. Traditionally, volume-averaged Euler-Euler methods have been employed for the analysis of PCM slurry flows [5-9]. In these methods, conservation equations are solved on a fixed grid and appropriate source terms, usually based on semi-empirical correlations, are introduced to account for mass, momentum and energy exchange between phases $[8,10]$. Recently, direct numerical simulation (DNS) of solid-liquid two-phase flows has gained increasing attention. DNS models do not rely on semi-empirical correlations to determine the interfacial forces and fluxes, rather these quantities are obtained by direct integration at the two-phase interface. Several DNS models have been proposed to investigate particulate flows by using either the fixed-mesh methods (e.g., the phase-field [11,12], level set [13], immersed boundary [14,15] and distributed Lagrange multiplier/fictitious domain 
methods [16])) or body-conformal mesh methods (e.g., the Arbitrary-Lagrangian-Eulerian (ALE) method) $[17,18]$ to directly solve the Navier-Stokes $[15,19,20]$ equations, as well as by solving the lattice Boltzmann equations [21,22]. Compared to the fixed-mesh methods, body-conformal mesh methods provide higher accuracy in interface representation; however, the higher accuracy comes at the expense of greater computational costs associated with mesh movement and re-meshing the computational domain. With the advancements in parallel computations on supercomputers/multi-core processors, this moving-mesh method is expected to become more computationally affordable.

The ALE method was developed to take advantage of the features of both the Lagrangian and Eulerian approaches, while minimizing their limitations [23]. In the ALE method, the mesh motion can be defined in an arbitrary manner with no constraint to follow a fix point of mass or to be fixed in space respectively [20,24]. Such a moving-deforming mesh allows for high-resolution tracking of boundary movements/deformation that is crucial in the analysis of particulate flows. In the ALE method, the movement of the mesh is usually constrained on the boundaries where it has to follow the particles and/or the confining flow boundary. Re-meshing is required to avoid large mesh distortions and data have to be mapped on to the new mesh for consecutive computations [25].

While numerous DNS studies of slurry flows have focused on the hydrodynamic interactions between the solid and fluid phases [26-30], there are only a few studies accounting for heat transfer and phase change effects [31-33]. Gan et al. [31] used a finite element implementation of the ALE method to study melting and sedimentation of one and two cylindrical particles in a vertical channel. Results were presented for instantaneous sedimentation velocity and mass of the particles. It was concluded that sedimentation of melting particles is affected by natural convection of the melt and that while two simultaneously settling particles maintain their circular shape during melting due to rotation, a single particle develops a non-circular surface morphology. Dierich et al. [32] used the implicit fictitious boundary method to simulate the phase change of up to 32 cylindrical ice particles ascending 
in a closed cavity, where the particles were assumed to remain circular during the phase change process. Three subsequent regimes of particle hydrodynamic were observed: acceleration of particles due to the buoyancy force, followed by a transitional regime and eventually a passive regime, where the solid particles are too small to influence the flow. In addition, a correlation was proposed between the Nusselt number at the surface of a single particle fixed in a hot stream and the Reynolds and Prandtl numbers. In a similar study, Dierich and Nikrityuk [33] analyzed the melting and solidification of 40 circular ice particles during upward motion in hot and subcooled water, respectively. It was found that during melting, the particles tend to move toward the center of the cavity while for solidification no significant displacement from the walls was observed. The effect of particle rotation was also investigated where about $10 \%$ increase in melting rate was observed when rotation of particles was accounted for while the solidification rate did not change significantly with and without particle rotation. Deen and Kuipers [34] applied the immersed boundary method to study the heat and mass transfer resulting from exothermal chemical reactions at the surface of stationary particles in a dense fluid-particle system. It was found that the average heat and mass transfer coefficients between the fluid and particles increase with the flow rate through the particle array, and the predictions from the DNS model were found in good agreement with the existing empirical correlations [34].

In addition to the gravity driven particulate flows with direct contact between the fluid and the phase change material (PCM), slurry flows with micro-/nano-encapsulated PCM particles have been investigated extensively [5,9,35-39]. In these studies, the encapsulated PCM slurry flows are often modeled as a homogenous fluid with average thermophysical properties using Eulerian methods on a fixed gird [5-9]. On the other hand, effective specific heat capacity [9] and enthalpy methods [40] have been introduced to account for solid-liquid phase change. In a relevant study, Rao et al. [39] experimentally studied the heat transfer of a micro-encapsulated PCM (MEPCM) slurry flow in minichannels, where $n$-octadecane MEPCM particles of about $5 \mu \mathrm{m}$ in diameter where suspended in 
water. Results showed an up to $40 \%$ increase in the average Nusselt number at the minichannel wall for 22.3 vol\% MEPCM. Kuravi et al. [9] developed a 3D numerical model to study the flow of nanoencapsulated PCM slurry in microchannels. The nano-encapsulated PCM slurry of $100 \mathrm{~nm}$ encapsulated octadecane particles suspended in polyalphaolefin was considered as a homogeneous fluid with average thermophysical properties. Melting of the PCM particles was accounted for using a specific heat capacity method in which a large specific heat value is adopted to mimic the phase change occurring over a melting temperature range. About $60 \%$ increase in the wall Nusselt number was reported for slurry with 30.3 vol\% PCM compared to the single-phase flow.

Review of the literature reveals that despite significant progress in modeling of PCM slurry flows using both DNS and volume-averaged methods, studies addressing non-uniform melting of multiple particles in slurry flows with simultaneous settling are scarce. In such slurry systems, the fluid motion is strongly influenced by the sedimentation of solid particles. In particular, the effect of simultaneous settling and melting of solid PCM particles on the heat transfer of slurry PCM flows with heating/cooling surfaces has not been addressed. Increasing interest in the utilization of solid-liquid phase change processes to improve the performance of thermal systems within a compact format leads to the emergence of PCM recirculation cycles where the PCM particles suspended in their own melt provide a short-term thermal storage between the hot and cold sides [41]. At the heat receiving side, a recirculating PCM cycle requires transfer of thermal load to a PCM slurry where thermal energy is absorbed by melting PCM particles. At the heat releasing side, PCM particles are then regenerated by the solidification process. For PCM particles of millimeters in size or larger, their body forces (e.g., buoyancy force due to density differences between the solid and liquid phases) play an important role on the hydrodynamic behavior of the slurry. Also, the deviations between the localized and average heat fluxes on the surface of individual particles increase with particle size. Detailed hydrodynamic and 
heat transfer analysis of such slurry flows using the DNS methods is hence crucial for the design of thermal systems utilizing PCM slurry flows.

In this work, a two-dimensional DNS model is developed to investigate simultaneous sedimentation and melting of multiple cylindrical PCM particles in their own melt in a vertical channel with heated walls. The main focus is on the heat transfer characteristics of the PCM slurry flow with the heating surfaces. An implementation of the ALE method in a finite volume formulation is employed. The melting rate of solid particles is determined from the localized heat fluxes at the particle surface. The model implementation is verified against published numerical results for simultaneous melting and settling of a single cylindrical particle. Improvements in heat transfer rates due to the presence of solid particles are quantified in terms of the average Nusselt number at the wall for varying particle volume fraction of the PCM slurry as compared to the single-phase case. The effects of the initial particle size and particle arrangement on the heat transfer characteristics of the slurry flow are also examined.

\section{Problem Formulation}

Slurry flow in a vertical channel accompanied by melting and settling of PCM particles in their own melt is considered in this work (Fig. 1). Solid PCM particles at constant melting temperature $T_{\mathrm{m}}$ are distributed throughout a channel filled with liquid PCM initially at $T_{\mathrm{m}}$. The channel walls are maintained at a constant temperature greater than $T_{\mathrm{m}}$. The solid particles initially at zero velocity settle under gravity and drag forces. A no-slip condition is applied at the side walls and a fully-developed velocity profile is applied at the top and bottom boundaries. Also, a fully-developed temperature profile is applied at the top boundary with a minimum value of $T=T_{\mathrm{m}}$ at the centerline along with an adiabatic condition at the bottom boundary. 


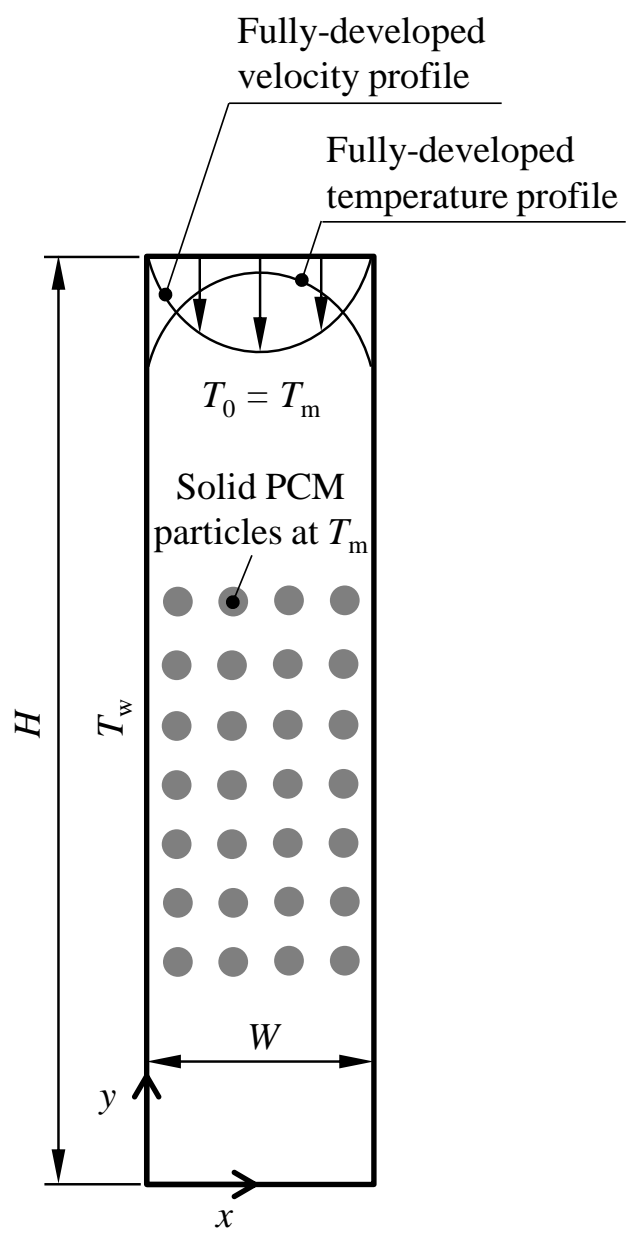

Fig. 1 Schematic of the physical system of multiple PCM particles simultaneously melting and settling in a vertical channel.

The computational domain encompasses the entire channel width with an aspect ratio (height to width) of 5.625 and contains a fixed number of circular particles. Initially stationary PCM particles accelerate under gravity, where the average instantaneous settling velocity of the particles in $y$ direction is calculated in the fixed frame of reference. The computational domain is then moved in each time step with the average settling velocity of the particles. The location of each particle within the moving computational domain is updated based on its relative translational velocity, where the $x$ component of the velocity is unchanged and the $y$-component is calculated by subtracting the domain velocity from the particle velocity in the fixed frame of reference. Moving the computational domain 
allows for reducing the domain size to a region with greatest likelihood of particle presence, while excluding the downstream and upstream regions of no particles. This transformation introduces an additional term in the $y$-momentum equation due to the acceleration of the reference frame $[42,43]$. Also, the $y$-component of the velocity at the boundaries in the transformed frame is obtained by subtracting the frame velocity from the absolute velocity at the boundary.

It is assumed in this work that the particles initially residing within the computational domain remain in it during the entire simulation time. To this end, if a particle reaches the top or bottom boundary, it is assumed that the particle sticks to the boundary as long as its calculated relative $y$ velocity is toward the exterior of the computational domain. The moving computational domain used here can be viewed as a section of a vertical channel at the top of which solid particles are loaded with a volume fraction equal to the initial volume fraction of the moving domain. The following assumptions are adopted in this work:

(i) The liquid phase is Newtonian whose thermophysical properties are assumed to be constant;

(ii) Natural convection is accounted for by the Boussinesq approximation;

(iii) Particle rotation is neglected;

(iv) Liquid flow induced by the volume change of the PCM upon melting is negligible; and

(v) Solid PCM particles are kept at $T_{\mathrm{m}}$.

\subsection{Governing equations}

The transient two-dimensional conservation equations for mass, momentum and energy are solved for the fluid phase in an Eulerian framework:

$$
\begin{gathered}
\nabla \cdot \mathbf{V}=0 \\
\frac{\partial \mathbf{V}}{\partial t}+\mathbf{V} \cdot \nabla \mathbf{V}=-\frac{1}{\rho_{l}} \nabla p+v \nabla^{2} \mathbf{V}+\left[1-\beta\left(T-T_{0}\right)\right] \mathbf{g}-\frac{\partial \mathbf{V}_{\text {frame }}}{\partial t}
\end{gathered}
$$




$$
\frac{\partial T}{\partial t}+\mathbf{V} \cdot \nabla T=\alpha \nabla^{2} T
$$

where $\rho_{l}$ is the density of the liquid phase at the initial temperature $T_{0}\left(T_{0}=T_{\mathrm{m}}\right), \beta$ the coefficient of thermal expansion, $p$ the pressure, $\alpha$ the thermal diffusivity, $v$ the kinematic viscosity, $\mathbf{g}$ the gravitational acceleration, and $\mathbf{V}$ the liquid velocity and $\mathbf{V}_{\text {frame }}$ the moving velocity of the reference frame. Again, $\mathbf{V}_{\text {frame }}$ in this work is equal to the average settling velocity of the particles calculated at each time step. It is noted that the last term on the right-hand side of Eq. (2) accounts for the acceleration of the reference frame [43]. The translational movement of the solid particles is governed by Newton's second law of motion:

$$
m_{i} \frac{d \mathbf{V}_{p, i}}{d t}=\mathbf{F}_{A, i}+\mathbf{F}_{d, i}
$$

where $m_{i}$ and $\mathbf{V}_{p, i}$ are the mass and absolute velocity of the $i^{\text {th }}$ particle and $\mathbf{F}_{A}$ and $\mathbf{F}_{d}$ are the Archimedes' and drag forces acting on the particle, following

$$
\begin{gathered}
\mathbf{F}_{d, i}=\oint_{S}\left[\mu\left(\nabla \mathbf{V}+\nabla \mathbf{V}^{T}\right) \cdot \mathbf{n}-p \mathbf{n}\right] d s \\
\mathbf{F}_{A, i}=\int_{V} \rho_{l}\left(1-\frac{\rho_{s}}{\rho_{l}}\right) \mathbf{g} d V
\end{gathered}
$$

where the integration in Eqs. (5) and (6) is performed over the particle surface area, $S$, and volume, $V$, respectively. The first and second terms of the integrand in Eq. (5) are the viscous stress and isotropic thermodynamic stress [44], respectively, where $\mu$ is the dynamic viscosity and $\mathbf{n}$ is the surface unit normal vector pointing out of the solid particle. Also, $\rho_{s}$ in Eq. (6) denotes the density of solid PCM. The local melting rate of the particle, $d \mathbf{r} / d t$, is determined by imposing an energy balance at the particle surface:

$$
\rho_{s} h_{s l} \frac{d \mathbf{r}}{d t}=-k_{l}(\nabla T \cdot \mathbf{n}) \mathbf{n}
$$


where $k_{l}$ is the liquid thermal conductivity, $h_{s l}$ is the PCM heat of fusion, and $\nabla T \cdot \mathbf{n}$ is the local temperature gradient normal to the particle boundary, respectively. Equation (7) assumes that the solid particle is kept at the melting point at all time.

In this work, the collision between the particles is simplified by assuming that all colliding particles move with the same velocity determined from the conservation of the linear momentum:

$$
\mathbf{V}_{p}^{\prime}=\frac{\sum m_{i} \mathbf{V}_{p, i}}{\sum m_{i}}
$$

with $\mathbf{V}_{p}^{\prime}$ being the velocity of the impacted particles after collision. It is noted that in order to prevent the formation of computational cells with a negative/zero volume, the direct contact between particles must be avoided during the simulation. This is done by introducing a critical distance between neighboring particles. When the distance between two particles is smaller than the critical value the particles are considered colliding. In this work, a critical distance of $4 d / 15$ is used to accommodate the boundary layer mesh around individual particles. A numerical experiment showed that reducing the critical distance to $d / 5$, with $d$ being the initial particle diameter, had no noticeable effect on the wall and particle heat transfer, but led to convergence difficulties. It is noted that, in the simulations, the particle boundary layer is usually consisted of 10 layers of non-uniform mesh with a growth rate of 1.2 and a minimum thickness of $0.01 \mathrm{~mm}$ in the vicinity of the particle boundary (i.e., the total thickness of $0.26 \mathrm{~mm})$.

The governing equations are non-dimensionalized for the sake of generality. The following dimensionless variables are defined: $\mathbf{V}^{*}=\mathbf{V} / u_{t e r}, x^{*}=x / d, t^{*}=t /\left(d / u_{t e r}\right), p^{*}=p /\left(v \rho_{l} u_{t e r} / d\right)$, $T^{*}=\left(T-T_{\mathrm{m}}\right) /\left(T_{\mathrm{w}}-T_{\mathrm{m}}\right), \mathbf{F}^{*}=\mathbf{F} /\left(v \rho_{l} L u_{t e r}\right)$, where $L$ is a unit length and $u_{t e r}$ is the terminal velocity of a non-melting single particle of diameter $d$ settling in an unbounded fluid. Also, $v$ and $T_{\mathrm{w}}$ are kinematic viscosity of the liquid and wall temperature, respectively. The terminal velocity $u_{t e r}$ is obtained from 
the corresponding Reynolds number that can be determined from a force balance between the buoyancy and drag forces acting on the settling cylinder:

$$
\frac{\pi d^{3} g}{2 \operatorname{Re}^{2} v^{2}}\left(\frac{\rho_{s}}{\rho_{l}}-1\right)=C_{D}=f(\operatorname{Re})
$$

It is noted that in the above equation, the characteristic length in the definition of Re number is the initial particle diameter. Replacing dimensional variables in Eqs. (1) - (4) and (7) with the above non-dimensional ones yields the following non-dimensional governing equations:

$$
\begin{gathered}
\nabla \cdot \mathbf{V}^{*}=0 \\
\operatorname{Re}\left(\frac{\partial \mathbf{V}^{*}}{\partial t^{*}}+\mathbf{V}^{*} \cdot \nabla \mathbf{V}^{*}\right)=-\nabla p^{*}+\nabla^{2} \mathbf{V}^{*}-\frac{\mathrm{Gr}}{\operatorname{Re}} T^{*} \mathbf{i}-\frac{\partial \mathbf{V}_{\text {frame }}^{*}}{\partial t^{*}} \\
\frac{\partial T^{*}}{\partial t^{*}}+\mathbf{V}^{*} \cdot \nabla T^{*}=\frac{1}{\operatorname{Re} \operatorname{Pr}} \nabla^{2} T^{*} \\
\frac{\pi}{4} \operatorname{Re}\left(\frac{\rho_{s}}{\rho_{l}}\right) m_{i}^{*} \frac{d \mathbf{V}_{p, i}^{*}}{d t^{*}}=\mathbf{F}_{A, i}^{*}+\mathbf{F}_{d, i}^{*} \\
\frac{1}{\operatorname{Ste}}\left(\frac{\rho_{s}}{\rho_{l}}\right) \frac{d \mathbf{r}^{*}}{d t^{*}}=-\frac{1}{\operatorname{Re} \operatorname{Pr}}\left(\nabla T^{*} \cdot \mathbf{n}\right) \mathbf{n}
\end{gathered}
$$

where $\mathbf{i}$ in Eq. (11) is the unit vector in the direction of the gravity. In formulation of Eqs. (11)-(14) the following dimensionless variables are employed; $\operatorname{Re}=u_{t e r} d / v, \operatorname{Pr}=v / \alpha, \mathrm{Gr}=g \beta \Delta T d^{3} / v^{2}, \operatorname{Ste}=k_{l}\left(T_{\mathrm{w}^{-}}\right.$ $\left.T_{\mathrm{m}}\right) /\left(\rho_{l} \alpha h_{s l}\right)$, where all properties are related to the liquid phase.

\section{Results}

The governing equations and associated boundary conditions are solved using the commercial CFD package ANSYS Fluent 15.0 [45]. The computational grid consists of up to 500,000 triangular and quadrilateral cells. The SIMPLE algorithm is used for coupling between pressure and velocity [46]. A boundary layer mesh is implemented around individual particles for more precise capturing of the 
heat fluxes and shear forces at the particle surfaces, both of which are used to determine the particle movement and surface morphology as described in Sec. 2.1. It is noted that in this work when the particle volume shrinks down to $4 \%$ of the initial value, the particle is assumed to no longer undergo phase change and become a flow tracer such that the boundary condition at the particle surface changes from constant temperature to adiabatic. In order to improve the numerical stability, the governing equations are solved using a two-step method, where the momentum equation is solved using the temperatures obtained from first solving the energy equation [47]. The movement of the particles is handled by a moving-deforming mesh that uses the Spring-Based Smoothing method [48]. After every time step, if the deformed mesh fails to satisfy the skewness or size criteria (skewness $<0.5,1 \times 10^{-5} \mathrm{~m}$ $<$ cell size $<10^{-4} \mathrm{~m}$ ), local re-meshing is performed to replace deteriorated cells with new ones. All simulations are performed using a time step of $5 \times 10^{-5} \mathrm{~s}$. Convergence criteria of $10^{-4}, 10^{-6}$ and $10^{-9}$ are used for continuity, momentum and energy equations, respectively.

\subsection{Model verification}

The model is verified by comparing the sedimentation velocity and melting rate of a single cylinder settling in its own melt in a vertical channel with the numerical results reported by Gan et al. [31]. As shown in Fig. 2, the melt is initially at thermal equilibrium with the wall when the particle is released at the channel centerline $10 d$ above the bottom boundary of the computational domain where $d$ is the initial particle diameter. Side and bottom boundaries are stationary and are maintained at a constant temperature $T_{\mathrm{w}}{ }^{*}=1$. The top wall is adiabatic with vanishing shear. The PCM particle is assumed to remain at the melting temperature $T_{\mathrm{m}}{ }^{*}=0$. Here, the characteristic velocity is equal to the maximum settling velocity of the particle that is not known a priori. The comparison is made for $\operatorname{Pr}=$ 0.7, $\operatorname{Re}=21.1, \mathrm{Gr}=100, \rho_{s} / \rho_{l}=1.00232$, and Ste $=0.0251$, where Re number is defined using the initial particle diameter and instantaneous velocity. As evident in Fig. 3a, the particle initially 
accelerates due to the dominant gravitational force. It later reaches a fairly constant velocity resulting from a balance between the gravitational and drag forces. At the later stage the particle decelerates as its mass reduces and drag forces (proportional to the surface area) overweigh the gravitational force (proportional to the volume). Figure $3 \mathrm{~b}$ shows that the melting rate gradually decreases with time, attributed to the decreasing heat transfer surface area at the particle surface. As shown in Fig. 3, excellent agreement between the results exists for both instantaneous Re number and normalized particle mass (defined as the ratio of instantaneous particle mass to its initial mass, $\mathrm{m} / \mathrm{m}_{0}$ ) with maximum deviations of $1 \%$ and $10 \%$, respectively, verifying the capability of the model to precisely capture the physical phenomena.

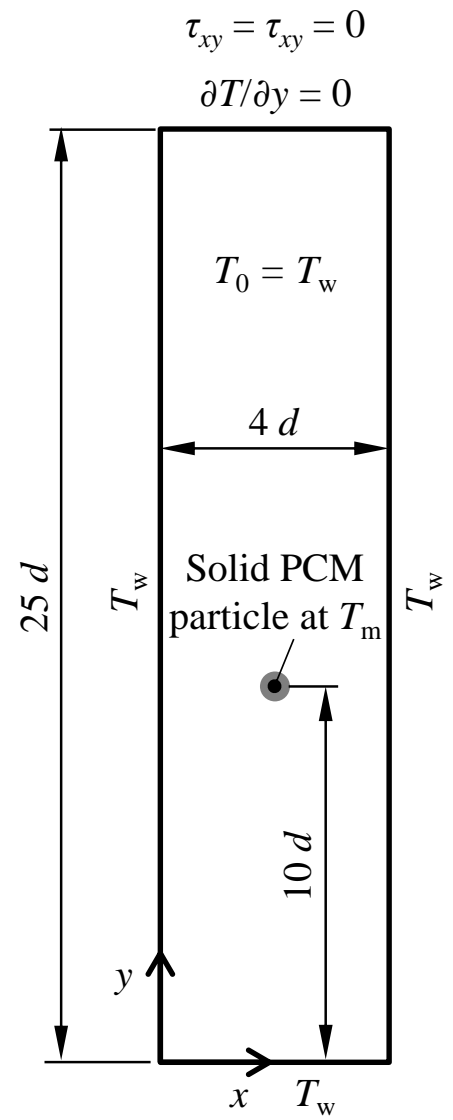

Fig. 2. Schematic of the physical system of a single cylindrical particle settling and melting in a vertical channel used for verification of the model. 

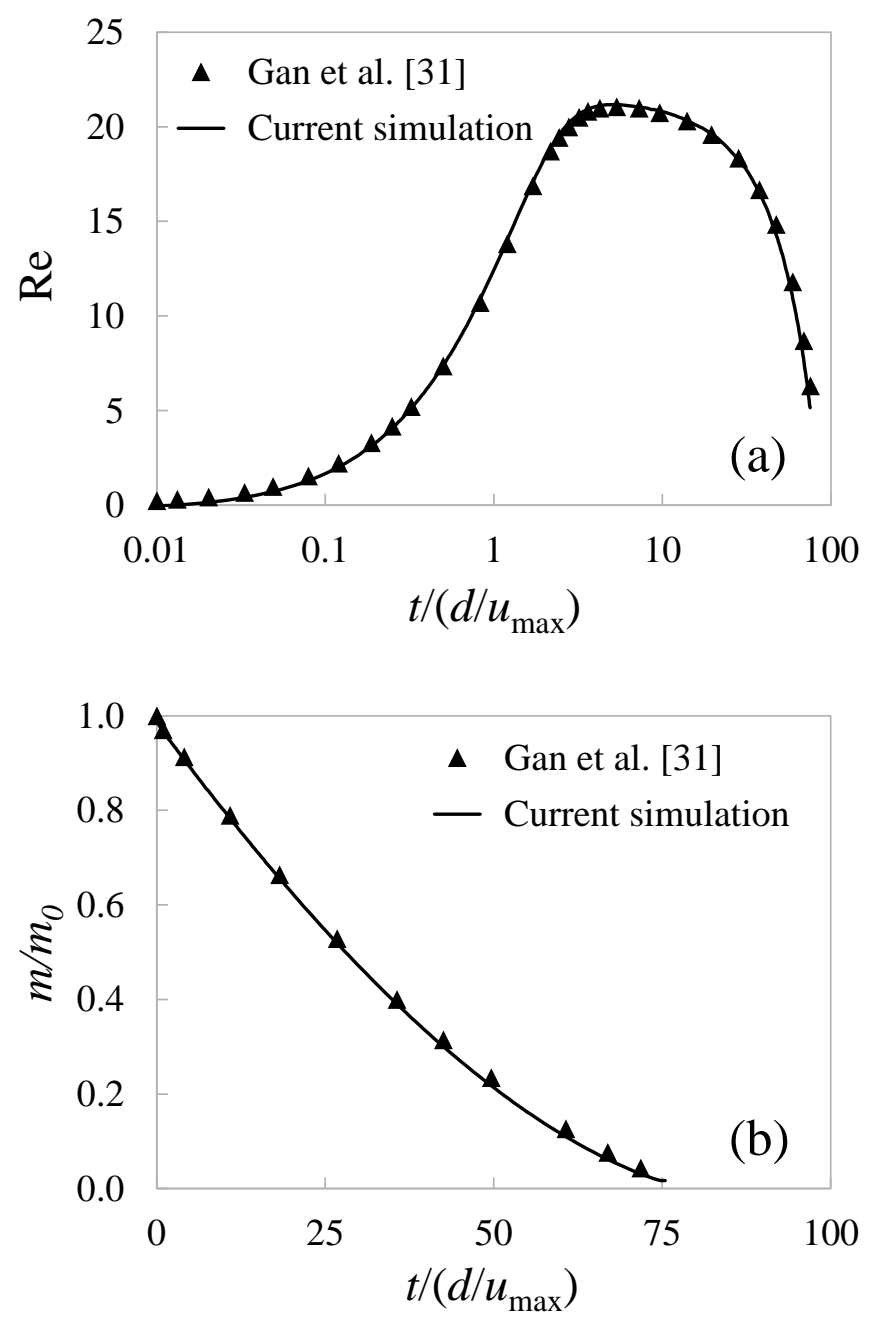

Fig. 3. Comparison of the time history of (a) instantaneous Re number defined using the initial particle diameter and instantaneous velocity and (b) normalized particle mass with the numerical results reported in [31].

\subsection{Case studies}

For the case studies, a vertical channel with height $H=0.18 \mathrm{~m}$ and width $W=0.032 \mathrm{~m}$ is considered (Fig. 1). The initial particle diameter is $0.003 \mathrm{~m}$ and the density ratio between the solid and liquid PCM is $\rho_{s} / \rho_{l}=1.00232$. Other thermophysical properties of the PCM are chosen to provide $\operatorname{Pr}=$ 0.7 , and Ste $=0.0251(\beta=0)$. Various particle numbers of $24,50,95$ and 150 are investigated to assess 
the effect of solid PCM volume fraction on the heat transfer behavior of the slurry (Fig. 4). These particle numbers correspond to volume fractions of $2.9 \%, 6.1 \%, 11.7 \%$ and $18.4 \%$, respectively, where the solid PCM volume fraction is calculated by dividing the initial solid PCM volume by the total volume of the channel.

In addition to the cases with an initial cylinder diameter of $0.003 \mathrm{~m}$, a case with 28 particles of $0.004 \mathrm{~m}$ initial diameter (corresponding to $6.1 \%$ initial solid PCM volume fraction) was also simulated to provide an insight on the effect of particle diameter on heat transfer for almost the same initial solid PCM volume fraction. In addition, the effect of initial particle arrangement inside the channel on heat transfer performance was evaluated by setting two different initial arrangements of 24 solid particles: one with an array of 6 rows and 4 columns (the 24-particle case in Fig. 4) and the other with 12 rows and 2 columns. The heat transfer rate between the PCM slurry flow and the wall is quantified by defining an average Nusselt number

$$
\mathrm{Nu}_{\mathrm{w}}=\frac{q_{\mathrm{w}, \mathrm{ave}}^{\prime \prime} D_{\mathrm{h}}}{T_{\mathrm{w}}-T_{\text {mean }}}
$$

where $q_{\mathrm{w}, \text { ave }}^{\prime \prime}$ is the average heat flux at the wall, $D_{\mathrm{h}}=2 W$ is the channel hydraulic diameter, and $T_{\text {mean }}$ is the mean flow temperature. For the PCM slurry flow, $T_{\text {mean }}$ is calculated by averaging the massweighted average flow temperature at several cross-sections, where flow velocities in the fixed frame of reference are used. It is noted that for a steady-state single-phase flow between two constant temperature parallel plates without natural convection effects, the energy equation can be solved analytically yielding $\mathrm{Nu}_{\mathrm{w}, \mathrm{ss}}=7.54$ [49]. A time-averaged $\mathrm{Nu}_{\mathrm{w}}$ can also be defined by averaging the $\mathrm{Nu}_{\mathrm{w}}$ from $t=0$ to the time when all the PCM particles are melted.

Numerical simulations showed that the time-averaged wall Nusselt number decreased almost 5\% by increasing the particle diameter by $33.3 \%$, which is considered negligible compared to $78 \%$ increase in the volume of individual particles. The time-averaged heat flux at the particle surface increased 1.36 
time by increasing the particle diameter. This increase is consistent with reduced total particle surface area associated with bigger particles (the total surface area of 28 particles of $0.004 \mathrm{~m}$ in diameter is $74 \%$ of that of 50 particles of $0.003 \mathrm{~m}$ in size). The change in the initial particle arrangement resulted in about 5\% difference in the time-averaged wall Nusselt number. These numerical simulations suggest that within the range of variations considered here, the particle size and initial arrangement do not have substantial effects on the overall heat transfer response of the slurry systems.

Figure 4 shows the initial arrangement of the particles for cases with particle numbers of 24, 50, 95 and 150 corresponding to cell counts of roughly 219,000, 222,000, 238,000, and 255,000, respectively. Grid independence study using $\sim 340,000$ cells ( $\sim 50 \%$ refined mesh) has shown less than $5 \%$ difference in calculated average wall Nusselt numbers for all four cases, verifying the sufficiency of the employed grid. It is noted that the cell count changes during simulations due to re-meshing process. According to the discussion above, the initial arrangement does not have a major effect on the overall thermal response of the slurry. Figure 5 shows the evolution of the temperature field throughout the domain for the case with 50 particles. The average temperature of the domain increases with time due to constant wall heating and reducing size of the solid particles that keep the average temperature from abrupt rising by latent heat of fusion. Inspection of the temperature fields at $t^{*}=50,233$ and 417 shows how the presence of solid particles in the vicinity of the wall breaks up the thermal boundary layer. This effect in addition to the mixing can contribute to the enhancement of the heat transfer in slurry flows. In addition to breakup of thermal boundary layer and mixing, PCM slurry flows also benefit from larger temperature differences that further improve the wall heat flux. The changes in distribution of particles can also be seen in Fig. 5, where no particular pattern can be identified and particles move in a random manner. It is noted again that the computational domain is moving with a velocity equal to the average sedimentation velocity of all the particles calculated at each time step. 
The relative location of computational domain at time instances corresponding to the displayed temperature fields is shown in the right panel of Fig. 5.

Figure 6 depicts the temperature field throughout the computational domain after the solid volume fraction has reduced to $50 \%$ of its initial value for the particle numbers of 24,50 , and 95 , corresponding to solid volume fractions of $1.45 \%, 3.05 \%$, and $5.85 \%$, respectively. The cases with fewer particles reach 50\% reduction of the initial solid PCM volume in a shorter time, mainly because of the smaller amount of solid PCM undergoing melting. Also, it can be seen in Fig. 6 that the particles do not melt uniformly, where the particles closer to the walls experience faster melting rates.

Figure 7 shows the time evolution of the wall Nusselt number (Fig. 7a) and the overall solid volume fraction (Fig. 7b) for slurry flows with 24, 50, and 95 particles of the same initial diameter of $0.003 \mathrm{~m}$. It is evident in Fig. $7 \mathrm{a}$ that the cases with greater solid volume fractions provide a larger $\mathrm{Nu}_{\mathrm{w}}$ over a longer time period. As discussed before, the greater Nusselt numbers corresponding to higher solid volume fractions can be explained by more effective heat transfer due to promoted mixing within the slurry flow and breakup of the thermal boundary layer due to a larger number of particles reaching the close vicinity of the wall. In Fig. 7a, the steady-state Nusselt number for a single-phase flow is also shown as a base case. Initially, the single-phase $\mathrm{Nu}_{\mathrm{w}}$ is more than two times greater than the steady state solution due to the absence of a thermal boundary layer and large temperature gradient at the heated wall in contact with an initially colder fluid. As time goes on, the fluid temperature adjacent to the wall increases and the temperature gradient at the wall decreases resulting in decreasing $\mathrm{Nu}_{\mathrm{w}}$ values. Eventually, the fully developed temperature field is established and $\mathrm{Nu}_{\mathrm{w}}$ approaches 7.54. It is noted that the heat transfer rates in PCM slurries with higher solid volume fractions also benefit from having greater temperature differences in addition to the improved heat transfer coefficients. Despite the noticeable fluctuations in the average Nusselt number versus time, the solid volume fractions 
reduce smoothly due to the damping effect of the thermal mass of the liquid that occupies the majority of the channel (Fig. 7b).

To better demonstrate the effect of melting solid particles in a slurry flow on the heat transfer rate of the heating surfaces, Fig. 8 shows the simulated wall Nusselt number as a function of the solid volume fraction for various particle loadings. It is noted that for each case in Fig. 8, the right end of the curve corresponds to the initial time of the simulation. It can be observed that the $\mathrm{Nu}_{\mathrm{w}}$ increases almost linearly with the solid PCM volume fraction. The melting PCM particles prevent the abrupt temperature rise of the slurry. Once the particles are completely melted, the fluid temperature increases rapidly and the heat flux at the wall decreases due to the reduced temperature difference between the heating surface and the fluid. The decreasing heat flux outweighs the reduction in temperature difference and the Nusselt number approaches the single-phase value. As a result, the $\mathrm{Nu}_{\mathrm{w}}$ for all cases drops rapidly around a solid volume fraction of zero. In Fig. 8, the steady-state single-phase Nusselt value is also shown as a base case. The gray dashed line in Fig. 8 is a linear curve fit through the data to show the linear trend of $\mathrm{Nu}_{\mathrm{w}}$ with solid fraction.

Another quantity of interest in the heat transfer analysis of the slurry flow is the heat transfer rates at the particle surface. The particle heat transfer rate is a representative of the rate of phase change and also can serve as an input for the mixture-based models that use correlations to account for interphase transport phenomena. Figure 9a shows the time evolution of the average Nusselt number at the particle surface for 24,50 , and 95 particles of the same initial diameter of $0.003 \mathrm{~m}$. To calculate the particle Nusselt number, the average heat flux at the particles' surface, $q_{\mathrm{p}, \text { ave }}^{\prime \prime}$, is first calculated and the value is then divided by the appropriate temperature difference to obtain an average heat transfer coefficient (here, $T_{\mathrm{w}}-T_{\mathrm{m}}$ is used). In addition, the average particle diameter, $d_{\mathrm{p}, \text { ave }}$, is calculated from the knowledge of the number of particles and their total volume as the characteristic length of the particle Nusselt number, following 


$$
\mathrm{Nu}_{\mathrm{p}}=\frac{q_{\mathrm{p}, \mathrm{ave}}^{\prime \prime} d_{\mathrm{p}, \mathrm{ave}}}{T_{\mathrm{w}}-T_{\mathrm{m}}}
$$

As shown in Fig. 9a, for the cases with smaller particle numbers (i.e. particle numbers of 24 and 50), there is an initial rise in the particle Nusselt number, $\mathrm{Nu}_{\mathrm{p}}$, followed by a gradual drop and eventually vanishing $\mathrm{Nu}_{\mathrm{p}}$. This initial rise can be attributed to the time needed for the heat to transfer from the heating wall to the particle surfaces due to the relatively large distance between the particles and the wall. As the time progresses, more heat reaches the particle surfaces, causing an increase in the particle Nusselt number. As the particles melt, the average particle diameter decreases. At the later stages of the process, the decrease in particle size exceeds the effect of increasing heat flux at the particle surface and the Nusselt number at the particle surface decays. For the case with 95 particles, the Nusselt number at the particle surface reduces more monotonically with time because the small distances between the closely arranged particles and the wall significantly shortens the initial time required for the heat to reach the particle surfaces. For this case, the particle Nusselt number gradually decreases due to shrinking solid particles. 


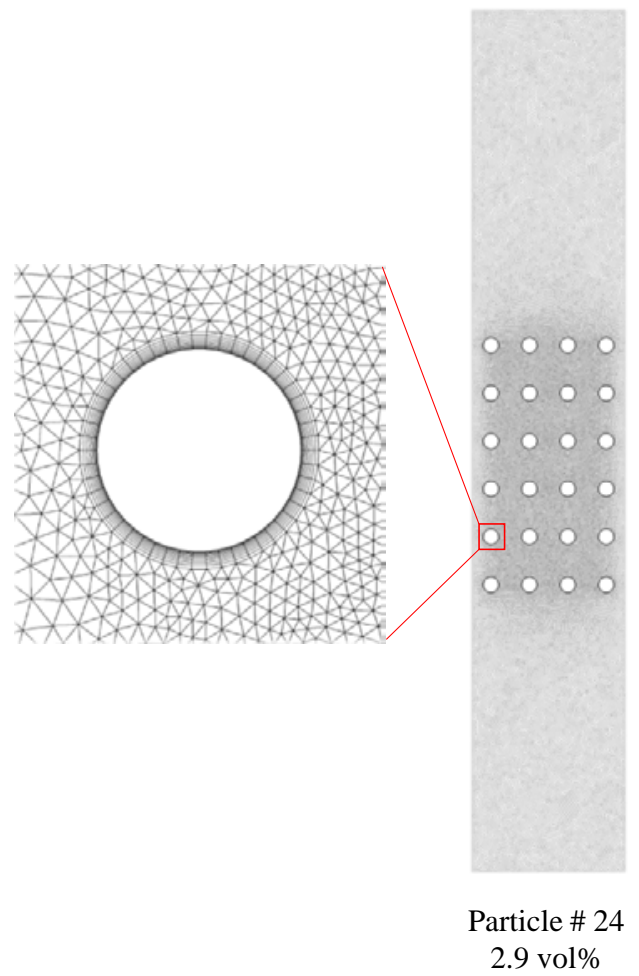

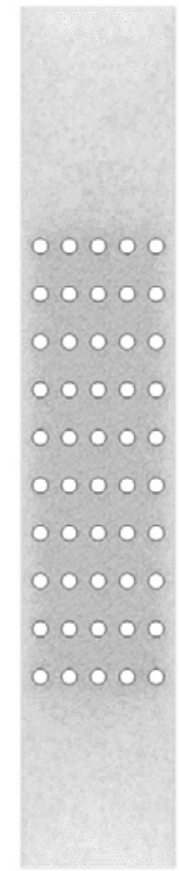

50

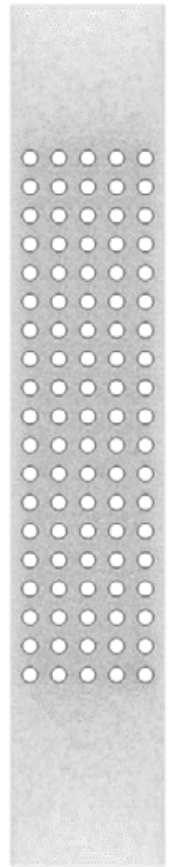

95

$11.7 \mathrm{vol} \%$

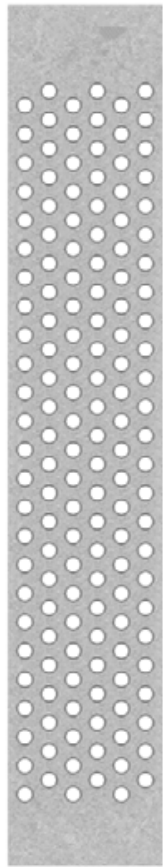

150

18.4 vol\%

Fig. 4. Initial mesh and distribution of solid PCM particles throughout the channel for various particle loadings but the same $0.003 \mathrm{~m}$ initial diameter, along with the zoomed-in view of the boundary layer mesh around a particle. 


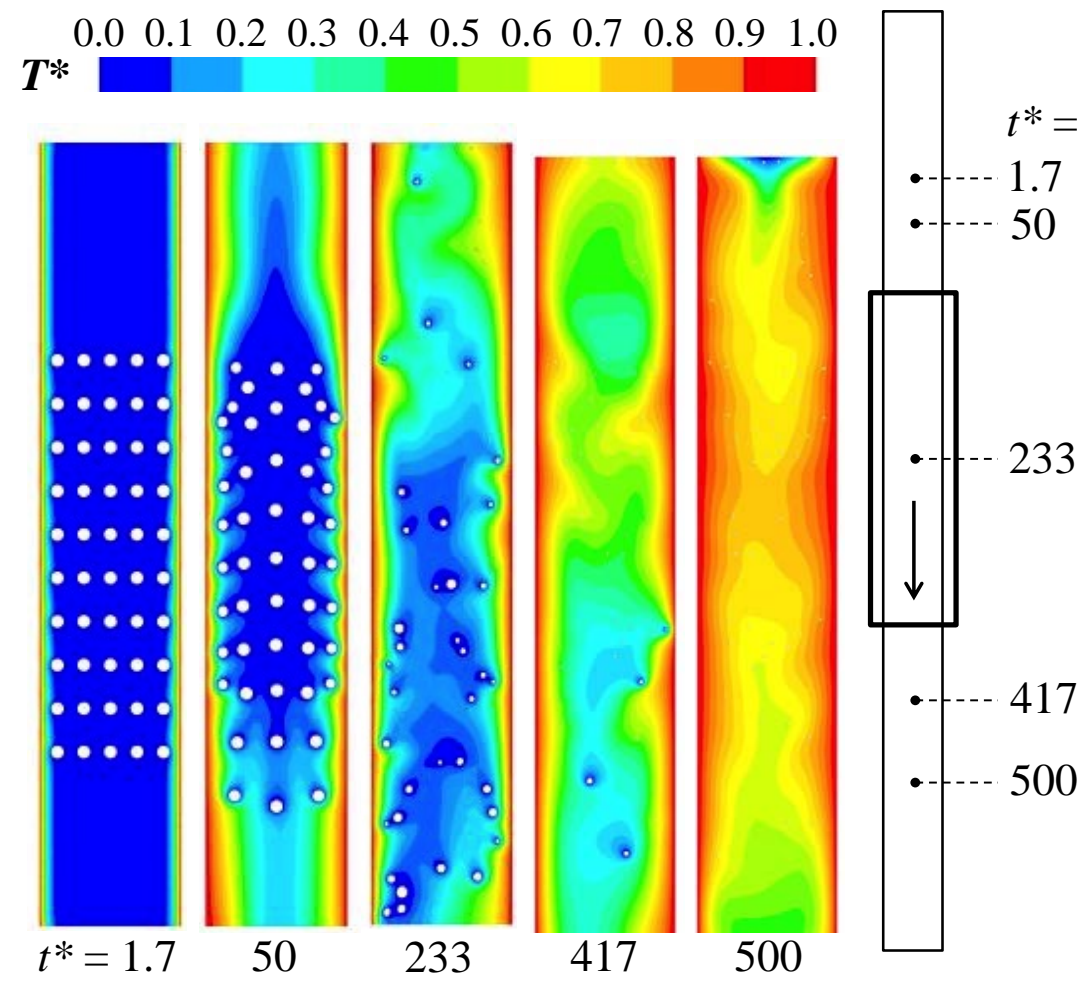

Fig. 5. Evolution of the temperature field throughout the computational domain for 50 particles of $0.003 \mathrm{~m}$ initial diameter. The right panel shows the relative location of the moving computational domain. 


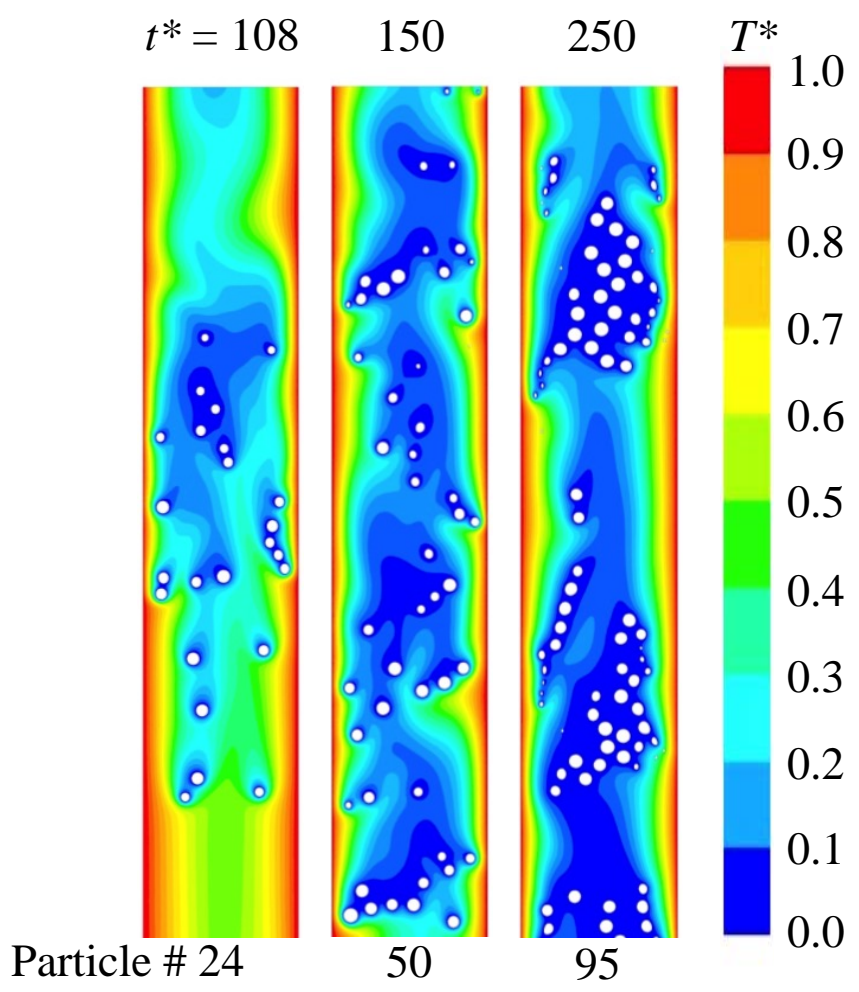

Fig. 6. Snapshots of temperature field for slurry flows with various particle loadings of the same initial diameter of $0.003 \mathrm{~m}$ at times corresponding to melting of half of the initial solid PCM. 

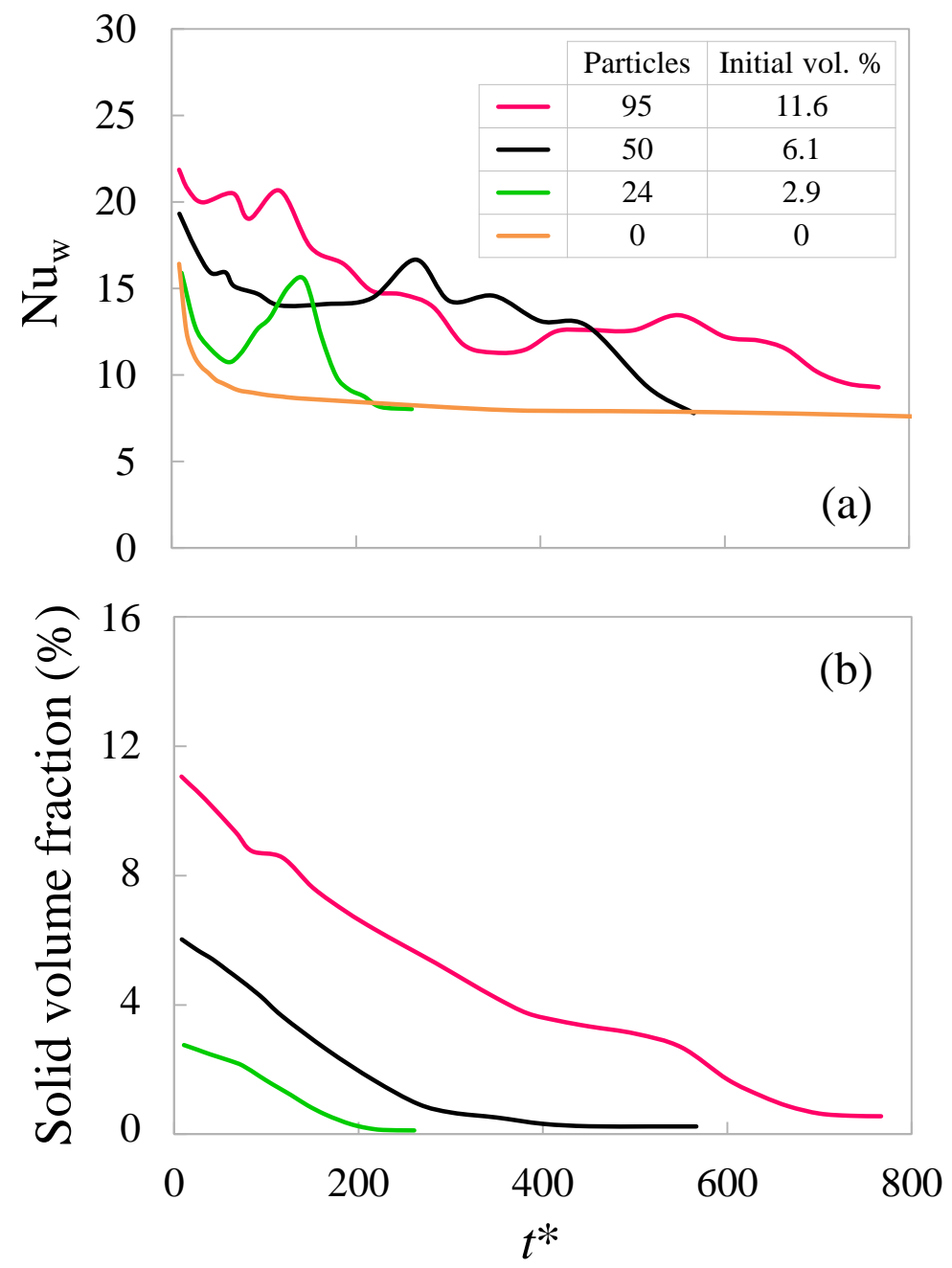

Fig. 7. Time evolution of (a) the average Nusselt number at the wall $(0.02 \mathrm{~m}<y<0.16 \mathrm{~m})$, and (b) solid PCM volume fraction for cases with the same initial particle diameter of $0.003 \mathrm{~m}$. 


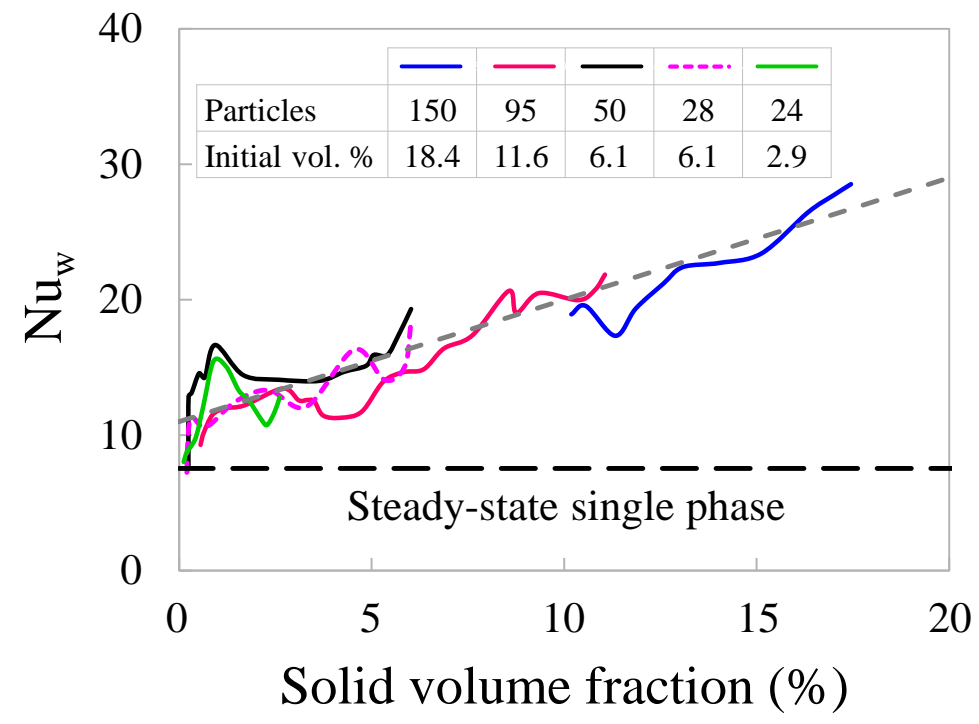

Fig. 8. The average wall Nusselt number versus solid PCM volume fraction for various particle loadings. The gray dashed line represents a linear curve fit through the data. 

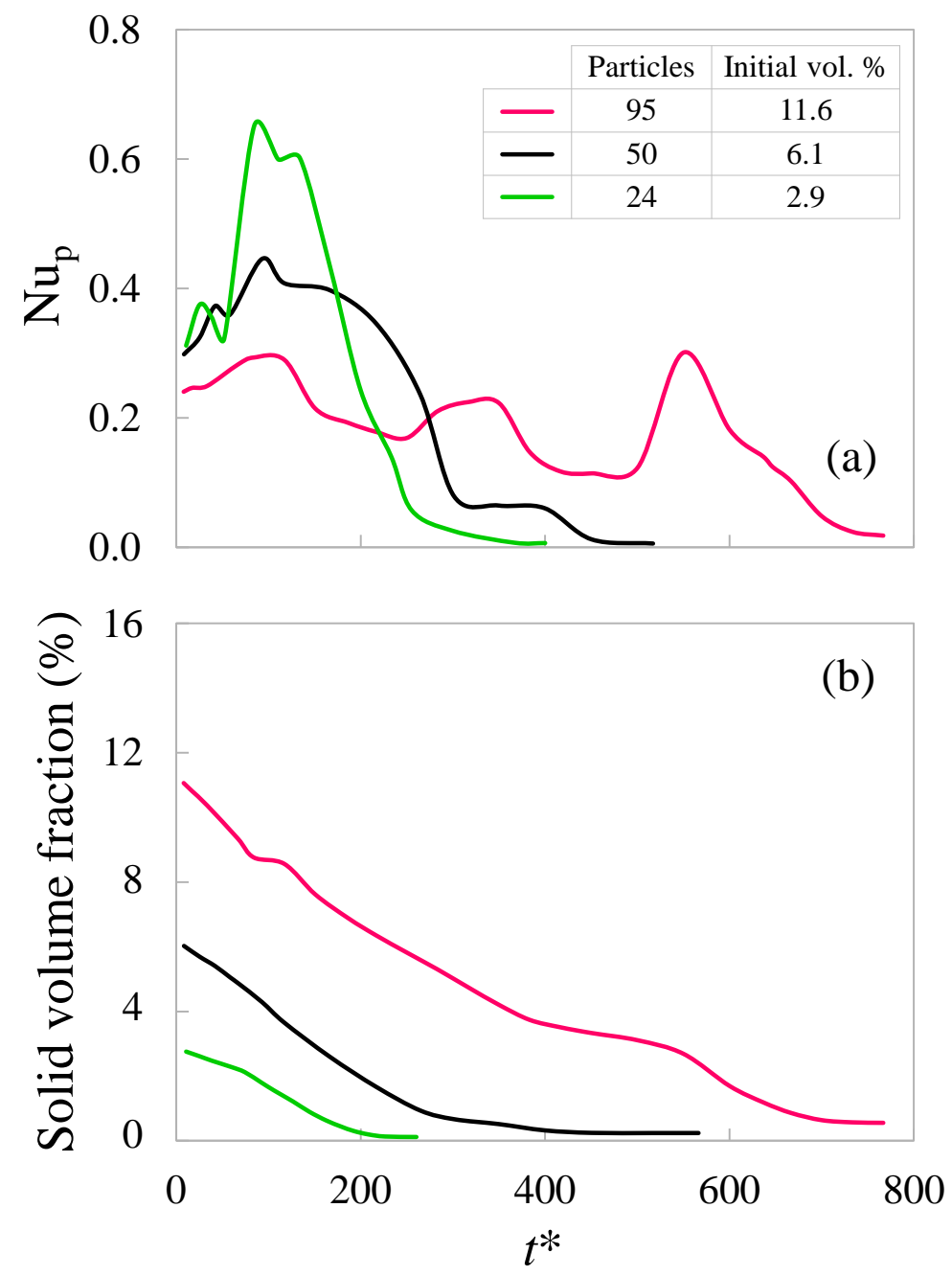

Fig. 9. Time variations of (a) average Nusselt number at the particle surface for particles of the same initial diameter defined by using $\Delta T=T_{\mathrm{w}}-\mathrm{T}_{\mathrm{m}}$ and (b) solid PCM volume fraction, for particles of the same initial diameter of $0.003 \mathrm{~m}$. 


\section{Conclusions}

Direct numerical simulation of slurry flows with simultaneous phase change and particle settling in a vertical channel with heated walls is carried out using the Arbitrary-Lagrangian-Eulerian method. Sedimentation and interface morphology changes of the particles are accounted for using a bodyconformal mesh. Average heat transfer rates at the wall and at the particle surfaces are presented in terms of average Nusselt numbers. It was found that the wall Nusselt number increases roughly linearly with solid PCM volume fraction, where more than a $100 \%$ increase over a single-phase flow is obtained at a solid fraction of $18 \%$. Additionally, it was found that the effects of initial particle arrangement are minimal and limited to only the early stages of heat transfer. Also the effects of particle diameter on the wall Nusselt number were found to be insignificant compared to the effect of solid volume fraction. The average Nusselt number at the particle surfaces was also determined, which can be used as an input to a less computationally expensive mixture-based Euler-Euler model for simulating slurry flows.

\section{Acknowledgment}

The financial support from the National Science Foundation (CBET-1357918) and The Electric

Power Research Institute (EPRI-10002061) is gratefully acknowledged.

\section{Nomenclature:}

$C_{D}$ : drag coefficient

F: force vector

Gr: Grashof number

g: gravitational acceleration vector

$H$ : height of the computational domain

$h_{s l}$ : PCM heat of fusion

i: unit vector in the direction of the gravity

$k$ : thermal conductivity 
$L$ : unit length

$m$ : particle mass

$\mathrm{Nu}$ : Nusselt Number

n: surface unit normal vector pointing out of the solid particle

$p:$ pressure

Pr: Prandtl number

Re: Reynolds number

r: solid-liquid interface coordinate vector

Ste: Stefan number

$T:$ temperature

$T_{\mathrm{m}}$ : melting temperature of PCM

$t$ : time

$u_{t e r}$ : terminal velocity of a settling non-melting particle

V: velocity vector

$W$ : width of the computational domain

$x$ : axis perpendicular to channel wall

$y$ : axis parallel to the channel wall

Greek letters:

$\alpha$ : thermal diffusivity of liquid PCM

$\beta$ : coefficient of thermal expansion

$\mu$ : dynamic viscosity

$v$ : kinematic viscosity

$\rho$ : density

\section{Subscripts:}

A: Archimedes

ave: average

$d$ : drag

frame: moving frame of the computational domain

$i$ : related to $i^{\text {th }}$ particle

$l$ : liquid PCM

$p$ : particle

$s$ : solid PCM

w: wall

0 : initial

Superscripts:

*: non-dimensional quantity

$\because$ quantity after collision 


\section{References}

[1] M. Delgado, A. Lázaro, J. Mazo, B. Zalba, Review on phase change material emulsions and microencapsulated phase change material slurries: Materials, heat transfer studies and applications, Renew. Sustain. Energy Rev. 16 (2012) 253-273.

[2] P. Zhang, Z.W. Ma, R.Z. Wang, An overview of phase change material slurries: MPCS and CHS, Renew. Sustain. Energy Rev. 14 (2010) 598-614. doi:10.1016/j.rser.2009.08.015.

[3] H. Inaba, New challenge in advanced thermal energy transportation using functionally thermal fluids, Int. J. Therm. Sci. 39 (2000) 991-1003. doi:10.1016/S1290-0729(00)01191-1.

[4] M. Delgado, A. Lázaro, J. Mazo, J.M. Marín, B. Zalba, Experimental analysis of a microencapsulated PCM slurry as thermal storage system and as heat transfer fluid in laminar flow, Appl. Therm. Eng. 36 (2012) 370-377. doi:10.1016/j.applthermaleng.2011.10.050.

[5] P. Charunyakorn, S. Sengupta, S.K. Roy, Forced convection heat transfer in microencapsulated phase change material slurries: flow in circular ducts, Int. J. Heat Mass Transf. 34 (1991) 819833. doi:10.1016/0017-9310(91)90128-2.

[6] Y. Zhang, A. Faghri, Analysis of forced convection heat transfer in microencapsulated phase change material suspensions, J. Thermophys. Heat Transf. 9 (1995) 727-732. doi:10.2514/3.731.

[7] X. Hu, Y. Zhang, Novel insight and numerical analysis of convective heat transfer enhancement with microencapsulated phase change material slurries: laminar flow in a circular tube with constant heat flux, Int. J. Heat Mass Transf. 45 (2002) 3163-3172. doi:10.1016/S00179310(02)00034-0.

[8] K.Q. Xing, Y.-X. Tao, Y.L. Hao, Performance evaluation of liquid flow with PCM particles in microchannels, J. Heat Transfer. 127 (2005) 931. doi:10.1115/1.1929783. 
[9] S. Kuravi, K.M. Kota, J. Du, L.C. Chow, Numerical investigation of flow and heat transfer performance of nano-encapsulated phase change material slurry in microchannels, J. Heat Transfer. 131 (2009) 062901. doi:10.1115/1.3084123.

[10] C. Beckermann, C.Y. Wang, Equiaxed dendritic solidification with convection: Part III. Comparisons with NH4Cl-H2O experiments, Metall. Mater. Trans. A. 27 (1996) 2784-2795. doi:10.1007/BF02652371.

[11] Y. Sun, C. Beckermann, Sharp interface tracking using the phase-field equation, J. Comput. Phys. 220 (2007) 626-653. doi:10.1016/j.jcp.2006.05.025.

[12] I. Steinbach, Phase-field models in materials science, Model. Simul. Mater. Sci. Eng. 17 (2009) 073001. doi:10.1088/0965-0393/17/7/073001.

[13] L.B. Tran, H.S. Udaykumar, A particle-level set-based sharp interface cartesian grid method for impact, penetration, and void collapse, J. Comput. Phys. 193 (2004) 469-510. doi:10.1016/j.jcp.2003.07.023.

[14] C.S. Peskin, Numerical analysis of blood flow in the heart, J. Comput. Phys. 25 (1977) $220-252$. doi:10.1016/0021-9991(77)90100-0.

[15] R. Mittal, G. Iaccarino, Immersed Boundary Methods, Annu. Rev. Fluid Mech. 37 (2005) 239261. doi:10.1146/annurev.fluid.37.061903.175743.

[16] R. Glowinski, T.-W. Pan, T.I. Hesla, D.D. Joseph, A distributed Lagrange multiplier/fictitious domain method for particulate flows, Int. J. Multiph. Flow. 25 (1999) 755-794. doi:10.1016/S0301-9322(98)00048-2.

[17] J. Donea, S. Giuliani, J.P. Halleux, An arbitrary lagrangian-eulerian finite element method for transient dynamic fluid-structure interactions, Comput. Methods Appl. Mech. Eng. 33 (1982) 689-723. doi:10.1016/0045-7825(82)90128-1. 
[18] H.H. Hu, Direct simulation of flows of solid-liquid mixtures, Int. J. Multiph. Flow. 22 (1996) 335-352. doi:10.1016/0301-9322(95)00068-2.

[19] R. Glowinski, T.W. Pan, T.I. Hesla, D.D. Joseph, J. Périaux, A Fictitious Domain Approach to the Direct Numerical Simulation of Incompressible Viscous Flow past Moving Rigid Bodies: Application to Particulate Flow, J. Comput. Phys. 169 (2001) 363-426. doi:10.1006/jcph.2000.6542.

[20] H.H. Hu, N.A. Patankar, M.Y. Zhu, Direct numerical simulations of fluid-solid systems using the Arbitrary Lagrangian-Eulerian technique, J. Comput. Phys. 169 (2001) 427-462. doi:10.1006/jcph.2000.6592.

[21] Z. Hashemi, O. Abouali, R. Kamali, Three dimensional thermal Lattice Boltzmann simulation of heating/cooling spheres falling in a Newtonian liquid, Int. J. Therm. Sci. 82 (2014) 23-33. doi:10.1016/j.ijthermalsci.2014.03.008.

[22] Z.-G. Feng, E.E. Michaelides, Robust treatment of no-slip boundary condition and velocity updating for the lattice-Boltzmann simulation of particulate flows, Comput. Fluids. 38 (2009) 370-381. doi:10.1016/j.compfluid.2008.04.013.

[23] H.H. Hu, D.D. Joseph, M.J. Crochet, Direct simulation of fluid particle motions, Theor. Comput. Fluid Dyn. 3 (1992) 285-306. doi:10.1007/BF00717645.

[24] J. Donea, A. Huerta, J.-P. Ponthot, A. Rodríguez-Ferran, Arbitrary Lagrangian-Eulerian Methods, in: E. Stein, R. de Borst, T.J.R. Hughes (Eds.), Encycl. Comput. Mech., John Wiley \& Sons, Ltd, Chichester, UK, 2004. doi:10.1002/0470091355.

[25] S. Haeri, J.S. Shrimpton, On the application of immersed boundary, fictitious domain and bodyconformal mesh methods to many particle multiphase flows, Int. J. Multiph. Flow. 40 (2012) 38-55. doi:10.1016/j.ijmultiphaseflow.2011.12.002. 
[26] A.J.C. Ladd, Numerical simulations of particulate suspensions via a discretized Boltzmann equation. Part 1. Theoretical foundation, J. Fluid Mech. 271 (2006) 285. doi:10.1017/S0022112094001771.

[27] D. Wan, S. Turek, Direct numerical simulation of particulate flow via multigrid FEM techniques and the fictitious boundary method, Int. J. Numer. Methods Fluids. 51 (2006) 531-566. doi:10.1002/fld.1129.

[28] N.A. Patankar, P. Singh, D.D. Joseph, R. Glowinski, T.-W. Pan, A new formulation of the distributed Lagrange multiplier/fictitious domain method for particulate flows, Int. J. Multiph. Flow. 26 (2000) 1509-1524. doi:10.1016/S0301-9322(99)00100-7.

[29] C.K. Aidun, Y. Lu, Lattice Boltzmann simulation of solid particles suspended in fluid, J. Stat. Phys. 81 (1995) 49-61. doi:10.1007/BF02179967.

[30] N.A. Patankar, D.D. Joseph, Modeling and numerical simulation of particulate flows by the Eulerian-Lagrangian approach, Int. J. Multiph. Flow. 27 (2001) 1659-1684. doi:10.1016/S03019322(01)00021-0.

[31] H. Gan, J.J. Feng, H.H. Hu, Simulation of the sedimentation of melting solid particles, Int. J. Multiph. Flow. 29 (2003) 751-769. doi:10.1016/S0301-9322(03)00035-1.

[32] F. Dierich, P. Nikrityuk, S. Ananiev, 2D modeling of moving particles with phase-change effect, Chem. Eng. Sci. (2011) 5459-5473.

[33] F. Dierich, P. Nikrityuk, Euler-Lagrange modelling of melting and solidification with moving solid particles, Prog. Comput. Fluid Dyn. 12 (2012) 112-118.

[34] N.G. Deen, J.A.M. Kuipers, Direct numerical simulation of fluid flow accompanied by coupled mass and heat transfer in dense fluid-particle systems, Chem. Eng. Sci. 116 (2014) 645-656. doi:10.1016/j.ces.2014.05.036. 
[35] B. Chen, X. Wang, Y. Zhang, H. Xu, R. Yang, Experimental research on laminar flow performance of phase change emulsion, Appl. Therm. Eng. 26 (2006) 1238-1245. doi:10.1016/j.applthermaleng.2005.10.040.

[36] E. Choi, Y. Cho, H. Lorsch, Forced-convection heat-transfer with phase-change-material slurries - turbulent-flow in a circular tube, Int. J. Heat Mass Transf. 37 (1994) 207-215.

[37] X.X. Wang, J. Niu, Y. Li, Y. Zhang, X.X. Wang, B. Chen, et al., Heat transfer of microencapsulated PCM slurry flow in a circular tube, AIChE J. 54 (2008) 1110-1120. doi:10.1002/aic.11431.

[38] Y. Yamagishi, H. Takeuchi, A.T. Pyatenko, N. Kayukawa, Characteristics of microencapsulated PCM slurry as a heat-transfer fluid, AIChE J. 45 (1999) 696-707. doi:10.1002/aic.690450405.

[39] Y. Rao, F. Dammel, P. Stephan, G. Lin, Convective heat transfer characteristics of microencapsulated phase change material suspensions in minichannels, Heat Mass Transf. 44 (2007) 175-186. doi:10.1007/s00231-007-0232-0.

[40] C.J. Ho, J.F. Lin, S.Y. Chiu, Heat transfer of solid-liquid phase-change material suspensions in circular pipes: effects of wall conduction, Numer. Heat Transf. Part A. (2010).

[41] Y. Sun, M. Mccarthy, Y.I. Cho, P. Boettcher, H. Hu, B. Shi, et al., Systems and methods of using phase change material in power plants, US patent 20150204612, 2015.

[42] D.J. Newman, G.E. Karnidakis, A direct numerical simulation study of flow past a freely vibrating cable, J. Fluid Mech. 344 (1997) 95-136. doi:10.1017/S002211209700582X.

[43] L. Li, S.J. Sherwin, P.W. Bearman, A moving frame of reference algorithm for fluid/structure interaction of rotating and translating bodies, Int. J. Numer. Methods Fluids. 38 (2002) 187-206. doi:10.1002/fld.216.

[44] A. Faghri, Y. Zhang, Transport Phenomena in Multiphase Systems, Academic Press, Elsevier, 2006. 
[45] FLUENT Inc., FLUENT 15.0 User Manual, (2015).

[46] S. Patankar, Numerical Heat Transfer and Fluid Flow (Hemisphere Series on Computational Methods in Mechanics and Thermal Science), McGraw-Hill, New York, 1980.

[47] S. Wang, A. Faghri, T.L. Bergman, A comprehensive numerical model for melting with natural convection, Int. J. Heat Mass Transf. 53 (2010) 1986-2000. doi:10.1016/j.ijheatmasstransfer.2009.12.057.

[48] J.T. Batina, Unsteady Euler airfoil solutions using unstructured dynamic meshes, AIAA J. 28 (1990) 1381-1388. doi:10.2514/3.25229.

[49] T.L. Bergman, A.S. Lavine, F.P. Incropera, D.P. DeWitt, Fundamentals of Heat and Mass Transfer, 7th ed., Wiley, Hoboken, 2011. 